\section{MATLAB-Based Educational Tool for Failure Analysis of Composite Laminates}

By: Ali, JSM (Ali, J. S. Mohamed) ${ }^{[1]}$; Mizuar, I (Mizuar, Iqtidar) ${ }^{[1]}$

\section{ADVANCES IN LIGHTWEIGHT MATERIALS AND STRUCTURES, ACALMS 2020}

Edited by: Kumar, AP; Dirgantara, T; Krishna, PV

Book Series: Springer Proceedings in Materials

Volume: 8 Pages: $807-816$

DOI: $10.1007 / 978-981-15-7827-4 \_82$

Published: 2020

Document Type: Proceedings Paper

\section{Conference}

Conference: 1st International Conference on Advanced Lightweight Materials and Structures (ICALMS)

Location: Hyderabad, INDIA

Date: MAR 06-07, 2020

Sponsor(s): CMR Grp Inst, Management

\section{Abstract}

An educational software has been developed for stress analysis of laminated composites. The software can be used to study both micro- and macro-mechanics analysis of a lamina as well as a laminate. The software can also be used for the failure analysis of laminates, and hence can be useful for the design of composite laminates for given loading conditions. Apart from mechanical loading, the software can also handle hygrothermal loading. Four different failure theories have been used to predict the failure of any given laminate. Graphical user interface (GUI) based on MATLAB language was used to develop this software, thus, making it more user-friendly. Numerous case studies were carried out and validated against the existing literature.

\section{Keywords}

Author Keywords: Failure; Composite; Hygrothermal; Software; Education

\section{Author Information}

Reprint Address:

International Islamic University Malaysia Int Islamic Univ Malaysia IIUM, Kulliyah Engn, Dept Mech Engn, Bangi, Selangor, Malaysia

Corresponding Address: Ali, JSM (corresponding author)

+ Int Islamic Univ Malaysia IIUM, Kulliyah Engn, Dept Mech Engn, Bangi, Selangor, Malaysia.

Addresses:

+ [ 1 ] Int Islamic Univ Malaysia IIUM, Kulliyah Engn, Dept Mech Engn, Bangi, Selangor, Malaysia

E-mail Addresses: jaffar@iium.edu.my

Funding

\begin{tabular}{|l|l|}
\hline Funding Agency & Grant Number \\
\hline Research Management Centre, International Islamic University Malaysia & \\
\hline
\end{tabular}

View funding text

Publisher

SPRINGER-VERLAG SINGAPORE PTE LTD, 152 BEACH ROAD, \#21-01/04 GATEWAYEAST, SINGAPORE, 189721,

SINGAPORE

\section{Categories / Classification}

Research Areas: Engineering; Materials Science; Transportation

Web of Science Categories: Engineering, Manufacturing; Engineering, Mechanical; Materials Science, Composites;

Transportation Science \& Technology

\section{See more data fields}

\section{Citation Network}

In Web of Science Core Collection

0

Times Cited

Create Citation Alert

8

Cited References

View Related Records

\section{Use in Web of Science}

Web of Science Usage Count

$\begin{array}{ll}0 & 0 \\ \text { Last } 180 \text { Days } & \text { Since } 2013 \\ \text { Learn more } & \end{array}$

This record is from:

Web of Science Core Collection

- Conference Proceedings Citation Index-

Science

Suggest a correction

If you would like to improve the quality of the data in this record, please suggest a correction. 


\section{Cited References: 8}

1. Educational Software for Stress Analysis of Idealized Thin Walled Open Sections

Times Cited: 1

By: Ali, J.S.M.; Fadhila, H.N.; Binti Aziz, N.A.

Applied Mechanics and Materials Volume: 315 Pages: 339-43 Published: 2013

2. Title: [not available]

Times Cited: 51

By: Datoo, $\mathrm{MH}$

Mechanics of fibrous composites Published: 1991

Publisher: Elsevier Applied Science, London

3. Title: [not available]

Times Cited: 330

By: Kaw, A.K.

Mechanics of composite materials Published: 2006

Publisher: CRC Press

4. A Software Tool for Mechanics of Composite Materials

Times Cited: 4

INTERNATIONAL JOURNAL OF ENGINEERING EDUCATION Volume: 13 Issue: 6 Special Issue: SI Pages: $433-441$ Published: 1997

5. LEADME: An Educational Software for Composite Laminate Analysis and Design

Times Cited: 1

Advanced Materials Research Volume: 1115 Pages: 578-81 Published: 2015

6. Educational software for stress analysis of non-idealized closed thin-walled sections

Times Cited: 1 By: Mohamed Ali, JS; Ibrahim, MOA; Mohammed, RM

Int J Eng Technol Volume: 7 Issue: 6 S Pages: 196-202 Published: 2019

7. MDSolids: Software to Bridge the Gap Between Lectures and Homework in Mechanics of Materials

Times Cited: 10 By: Philpot, Timothy A.

INTERNATIONAL JOURNAL OF ENGINEERING EDUCATION Volume: 16 Issue: 5 Special Issue: SI Pages: $401-407$ Published: 2000

8. Title: [not available]

Times Cited: 24

By: Voyiadjis, GZ; Kattan, PI.

Mechanics of composite materials with matllab Published: 2005

Publisher: Springer, Berlin

Showing 8 of 8 View All in Cited References page 